\title{
Optical Studies on Methyl Methacrylate Polymerized via Metal Carbazone Complexes
}

\author{
M. A. El-Sherbiny* and El-Said H.El-Mosallamy \\ * Physics Department, Faculty of Science, Al-Azhar University, Cairo, \\ (EGYPT) \\ Chemistry Department, Faculty of Science, Ain Shams University, Cairo, \\ (EGYPT) \\ E. Mail: sherbinypsi@yahoo.com
}

Optical studies are done on Poly methyl-methacrylate (PMMA) sheets. Samples were previously prepared by catalytic chain transfer polymerization via metal carbazone of Cd(II) and Hg(II). Transmission and absorbance are recorded (between $190 \mathrm{~nm}$ to $1100 \mathrm{~nm}$ ) as a function of incident energy using UV-VIS spectrophotometer. Second derivative of absorbance spectra is calculated to enhance differences among spectra. The optical band gap, width of band tail and dispersion of refractive index of the sheets are determined.

\section{Introduction}

Poly methyl-methacrylate (PMMA) (acrylic or plexiglass) often used as a thermoplastic glass. It is characterized by its high transparency and low birefringence [1], and possessing reasonably good environmental stability and good workability [2].

PMMA is frequently used as a substrate material for precision optics components [3] such as an optical switch, coupler, splitter and a transceiver [4], in memory materials [5], gas sensing [6], can be tailored chemically to fit wide range of photonics and optoelectronics applications ${ }^{7}$ and liquid crystal display [8]. For easy flow in polymer processing, low molecular weight chains are required $[9,10]$. This molecular weight reduction can be achieved by presence of organometallic complexes during the polymerization processes.

In recent work [11] poly methyl methacrylate was synthesized by catalytic chain transfer polymerization via metal carbazone of $\mathrm{Cd}(\mathrm{II})$ and $\mathrm{Hg}(\mathrm{II})$. Gradual increase of conversion\% was previously recorded with increasing complex concentration till maximum activity at $1.18 \times 10^{-4}$ and 1.86 
$\mathrm{x} 10^{-4} \mathrm{~g} \mathrm{~mol} / \mathrm{L}$ of $\mathrm{Cd}$ and $\mathrm{Hg}$ crbazone complexes. all chain transfer mechanisms involve abstraction of a hydrogen atom.

In the same work, X-ray showed a high relative degree of crystallinity at low complex concentrations was found to decrease as the concentrations increased.

FTIR reveals disappearance of a doublet band at 2353-2312 $\mathrm{cm}^{-1}$ corresponding to $\mathrm{N}=\mathrm{C}-\mathrm{N}=\mathrm{N}$, in addition to raising of two weak bands at 1000 $\mathrm{cm}^{-1}$ and $820 \mathrm{~cm}^{-1}$ assigned for $\mathrm{CH}_{2}-\mathrm{N}$ stretching vibration with addition of complex carbazone, these two bands were attributed to the hemolytic rapture of $\mathrm{N}=\mathrm{N}$ and $\mathrm{N}-\mathrm{H}$ bonds of the complex compound by the radical formed in the medium during polymerization process. These bonds rupturing might led to formation of active centers on the complex compound. Molecular weights, numbers and polydispersity reduction was recorded with increasing complex concentration.

The present work aims to study the effect of using $\mathrm{Cd}$ and $\mathrm{Hg}$ carbazone complex as an additive through the polymerization of methylmethacrylate on the optical properties of poly methylmethacrylate sheets

\section{Experimental}

Transparent films of Poly methyl methacrylate with a thickness of $\sim 1 \mathrm{~nm}$ were prepared by catalytic chain transfer via metal carbazone of $\mathrm{Cd}(\mathrm{II})$ and $\mathrm{Hg}(\mathrm{II})$ with deferent concentrations. Details of polymerization process and film formation was discussed else where [11].

In the present work both transmission and optical absorption spectra were recorded for the prepared polymer sheets using a Genway 6405 -UV-VIS spectrophotometer in the range $190 \mathrm{~nm}$ to $1100 \mathrm{~nm}$.

\section{Results and Discussion}

\subsection{Transmittance and Absorbance spectra.}

Transmission spectra of the methyl methacrylate polymerized in absence and presence of $\mathrm{Cd}$ and $\mathrm{Hg}$ carbazone with different concentrations (2.37, 7.11, $11.85,16.58,21.32\left(\mathrm{x} 10^{-5} \mathrm{~g} . \mathrm{mol} / \mathrm{L}\right)$ for $\mathrm{Cd}$ and $2.65,7.95,13.25,18.55,23.85$ $\left(\mathrm{x} 10^{-5} \mathrm{~g} . \mathrm{mol} / \mathrm{L}\right.$ ) for $\mathrm{Hg}$ ), have been measured in the spectral range $190 \mathrm{~nm}$ to $1100 \mathrm{~nm}$ were presented in Fig. (1). Pure PMMA shows high transparency with average transmittance above $80 \%$, with increasing $\mathrm{Cd}$ concentration, the transmittance reduced to $50 \%$ up to the concentration $16.58 \times 10^{-5} \mathrm{~g} \cdot \mathrm{mol} / \mathrm{L}$ then 
the sample retains transmittance about $80 \%$ again at higher concentrations. From the figure. It is observed that the edge exhibits red shift by increasing the $\mathrm{Cd}$ carbazone content up to $\sim 7.11 \times 10^{-5}$ g.mol/L. At higher $\mathrm{Cd}$ contents $\left(>7.11 \times 10^{-5}\right.$ g.mol/L) it shows an opposite trend, where it shows blue shift up to the highest concentration.

Polymerized samples with $\mathrm{Hg}$ carbazone showed the same conversion behavior in spite of the fact that all samples showed transmittance ranging between 85 and $75 \%$. In this case, the transparency of the sample fell to $75 \%$ at the first added concentration of mercury $2.37 \times 10^{-5}$ g.mol/L and then began to increase gradually with increasing concentration until it reached $85 \%$ again.
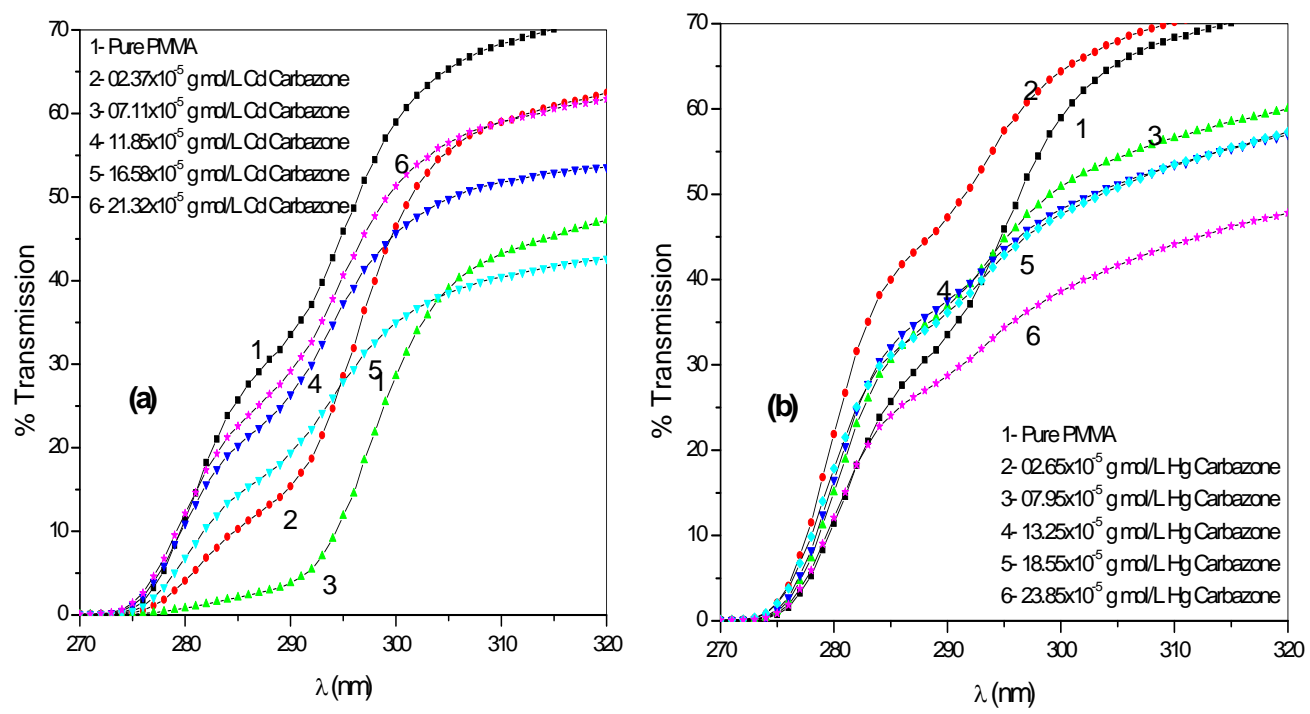

Fig. (1): Transmittance spectra of the methyl methacrylate polymerized in absence and presence of different concentrations of $\mathrm{Cd}$ (a) and $\mathrm{Hg}$ (b) carbazone.

The spectra in Fig. (2) illustrates the dependence of absorption on wavelength, Optical absorption edge in UV region broadened and exhibit red shift with addition of metal complex up to a certain concentration, and fluctuates with increasing concentration above this value. These observations indicates the conjugation of bonds in this region. 

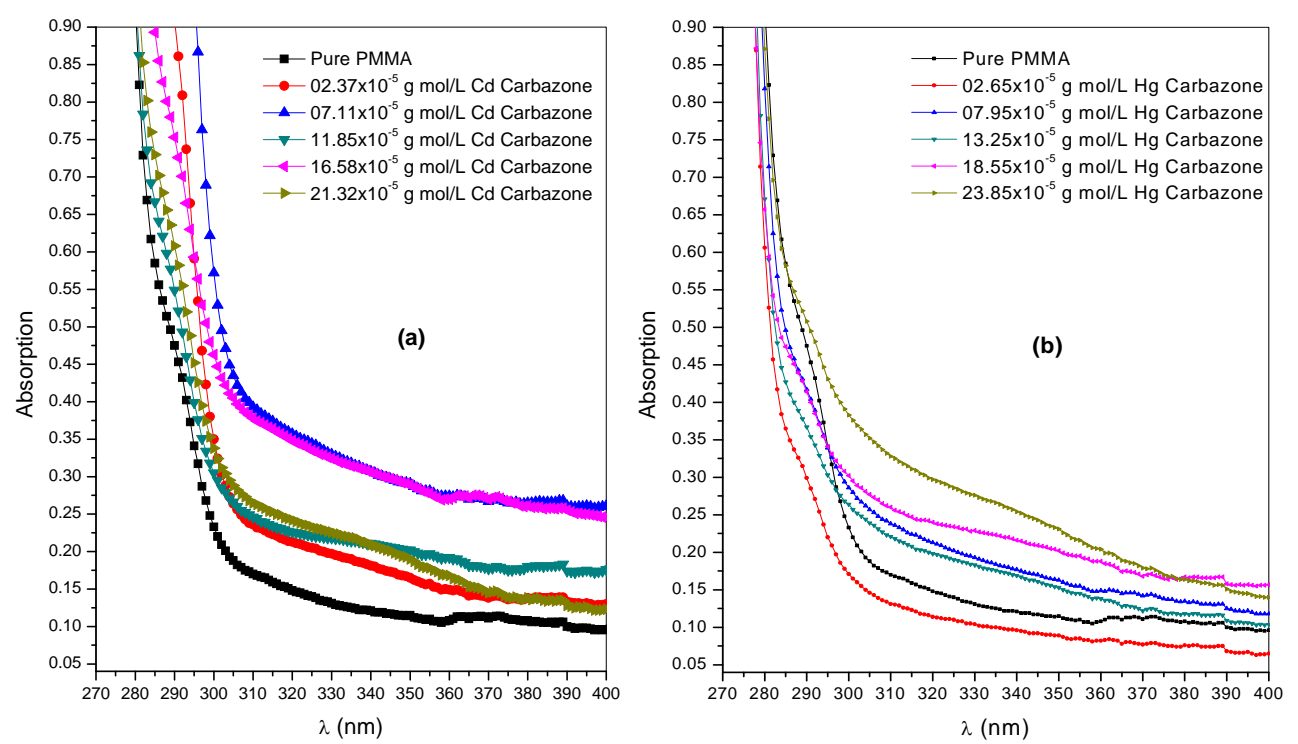

Fig. (2): UV-VIS spectra of the methyl methacrylate polymerized in absence and presence of different concentrations of $\mathrm{Cd}$ (a) and $\mathrm{Hg}$ (b) carbazone.

To enhance differences among spectra, second derivative spectra was calculated and presented in Fig. (3). Two peaks were observed and assigned to $\pi \rightarrow \pi^{*}$ transition located at $283 \mathrm{~nm}$, and $n \rightarrow \pi^{*}$ transition at $300 \mathrm{~nm}$. For accurate determination of the peak position, peaks were fitted with Amplitude Version of Gaussian function according to the relation:

$$
y^{u t}=y_{0}+A e^{-\frac{\left(s+\delta y^{2}\right.}{2 F^{2}}}
$$

where $y^{/ /}$is the second derivative of absorbance, $y_{0}$ is the offset, $w$ is the peak half width, and $\lambda_{c}$ is the center of the peak. The most distinctive feature of the second-order derivative is a negative band with minimum at the same wavelength as the maximum on the zero-order band [12].

A decrease of intensity of the $\pi \rightarrow \pi^{*}$ absorption peak with adding $\mathrm{Cd}$ carbazone is observed up to $7.11 \times 10^{-5} \mathrm{~g} . \mathrm{mol} / \mathrm{L}$ concentration. Further increase in Cd concentration, the $\pi \rightarrow \pi^{*}$ absorption peak began to appear again. The observed behavior can be accounted for by considering the delocalization of $\pi$ electrons. The later play the principle rule in determining the optical behavior of 
the polymer. The observed red shift is most likely due to the increase of $\pi$ electron delocalization resulting from the increase of chain length during the polymerization process. This in fare agreement with the data given in El-Mosallamy [11], where the number of reacting monomers increased and then start to decrease.

The estimated values of optical band gap follow the same trend, where it decreases then start to increase and approach the value of the MMA polymerized in absence of $\mathrm{Cd}$ carbazone. In this respect one should expect that the probability of lamella formation increases by increasing the chain length. The formation of the lamella means the increase of the degree of disorder, which explain the increase of the band tail width $\left(\mathrm{E}_{\mathrm{U}}\right)$ (Fig. 9.b).

The above mechanism ceases by the formation of dead polymer chains, where the reactive centers are destructed, which occurs by the bimolecular reaction between radicals, or by dispropotionation, in which a hydrogen radical that is beta to one radical center is transferred to another radical center. Increasing $\mathrm{Cd}$ concentration above $7.11 \times 10^{-5} \mathrm{~g} \cdot \mathrm{mol} / \mathrm{L}$, new active centers may be formed as a result of rupturing of $\mathrm{N}=\mathrm{N}$ and $\mathrm{N}-\mathrm{H}$ bonds of the complex by the radicals. Excitation of an electron from one of the unshared pair to the antibonding $\pi$ orbital can be noticed from the peak indicated $n \rightarrow \pi *$ which did not show a significant change with increasing the concentration of the complex.

The dependence of peak position of both $\pi \rightarrow \pi^{*}$ and $n \rightarrow \pi *$ transitions is shown in Fig. (4). It is observed that both transitions show opposite behavior in case of using $\mathrm{Cd}$ carbazone. However, in case of $\mathrm{Hg}$ they follow the same pattern. This can be related to the stability of the $6 s$ shell of $\mathrm{Hg}$ due to the presence of a filled $4 f$ shell, which screens the nuclear charge and resist the removal of an electron. 

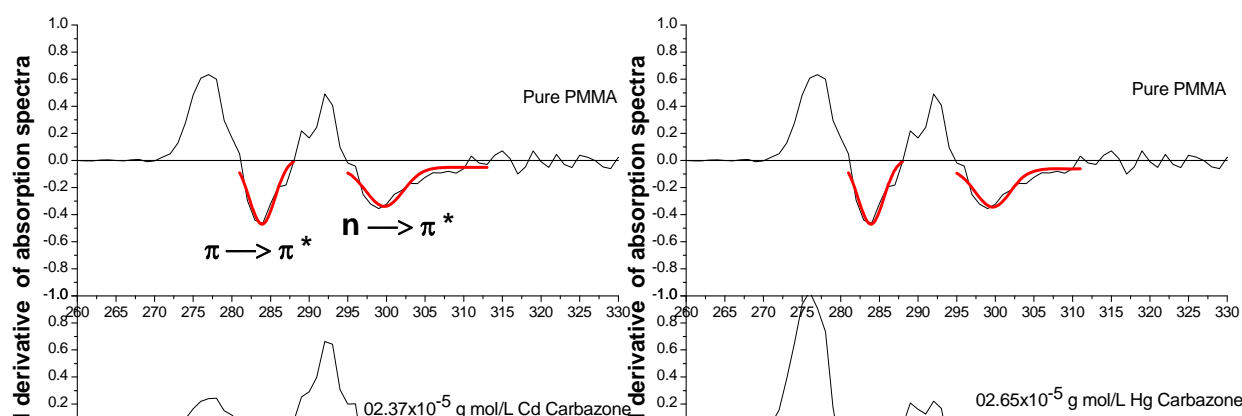

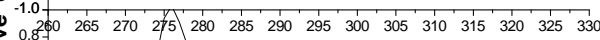
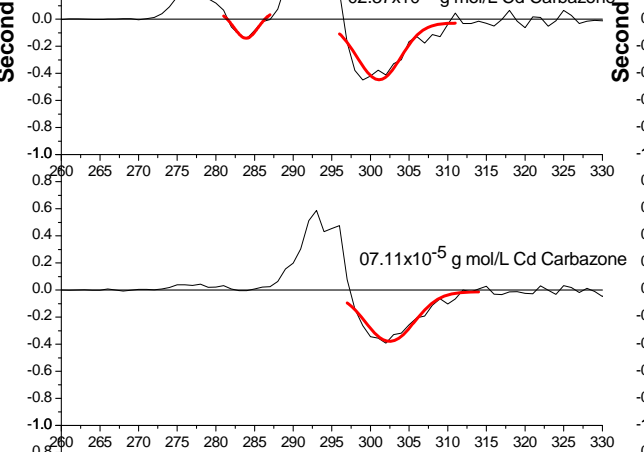

0.6
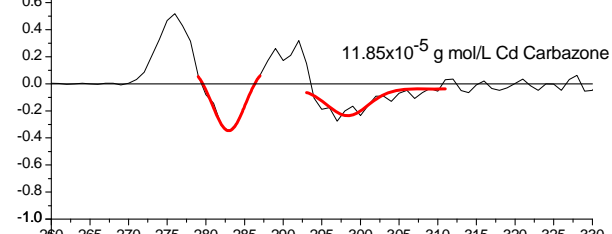

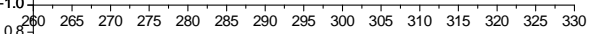

0.6
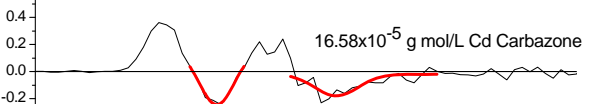

$0.4-$

-0.6
$-0.8-$
-1.0

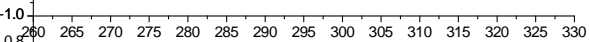

0.6

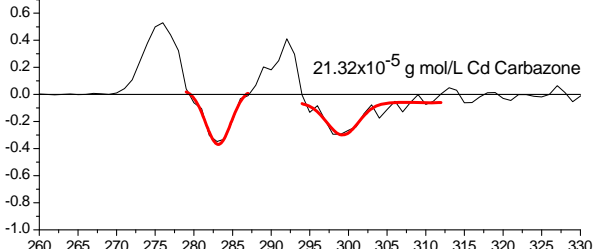

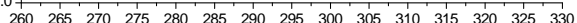
Wavelength $\lambda(\mathrm{nm})$

$02.65 \times 10^{-5} \mathrm{~g} \mathrm{~mol} / \mathrm{L} \mathrm{Hg}$ Carbazone

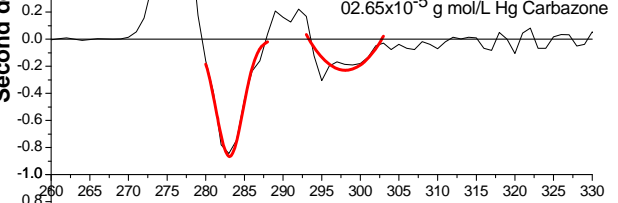

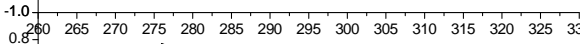
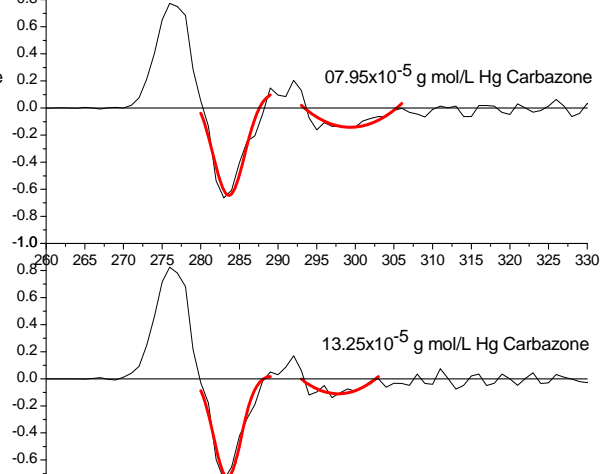

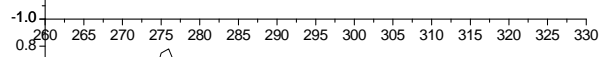
$18.55 \times 10^{-5} \mathrm{~g} \mathrm{~mol} / \mathrm{L} \mathrm{Hg}$ Carbazone

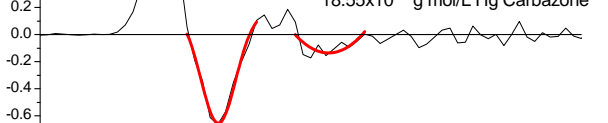

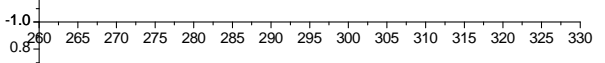

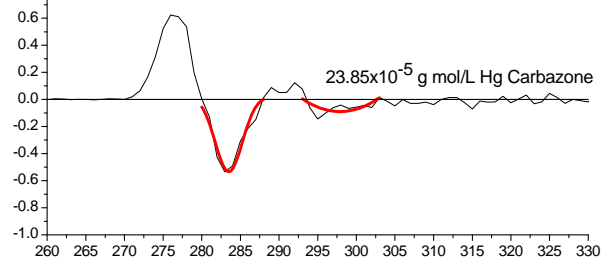
Wavelength $\lambda(\mathrm{nm})$

Fig. (3): second derivative of absorbance spectra for PMMA and metal complex polymerized MMA sheets 

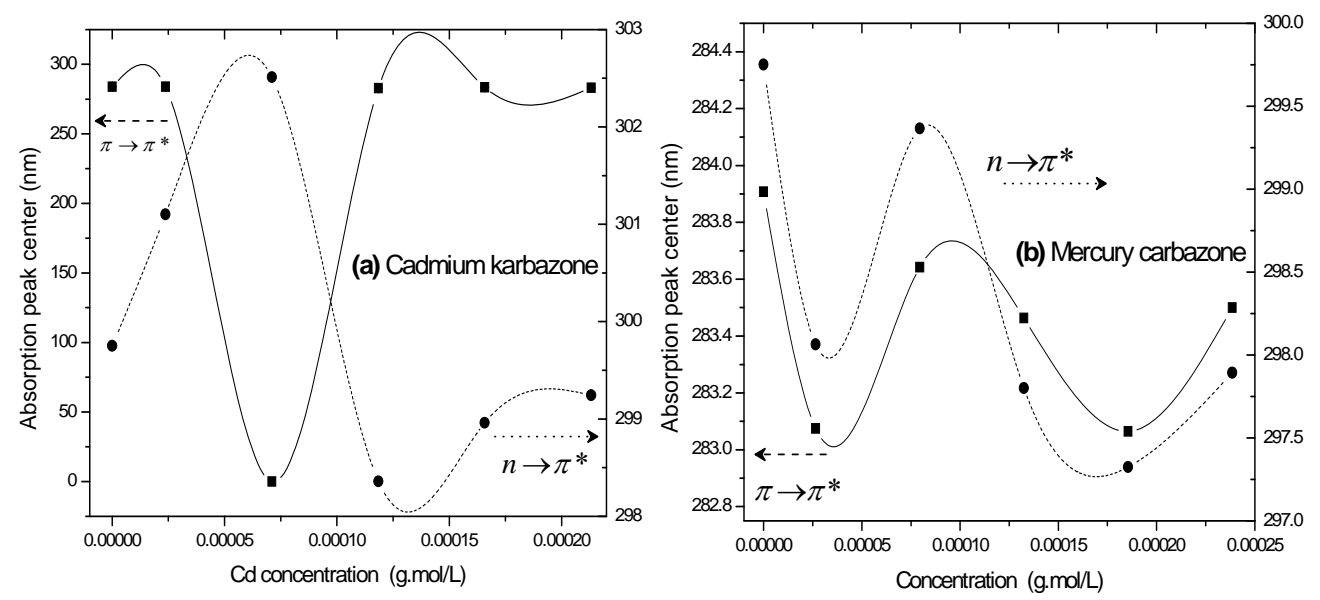

Fig. (4): Center of the absorption peaks as a function of $\mathrm{Cd}$ (a) and $\mathrm{Hg}$ (b) concentration (g.mol/L).

The optical absorption coefficient $\alpha(v)$ was calculated and presented in Fig. (5) through the well known relation [13].

$$
\alpha(v)=\frac{2.303}{d} \log \left(\frac{I_{0}}{I}\right)=\frac{2.303}{d} A(v)
$$

where $\mathrm{I}_{0}$ and $\mathrm{I}$ are the incident and transmitted intensities respectively, $\mathrm{d}$ is the sample thickness and $A(v)$ is the optical absorbance.
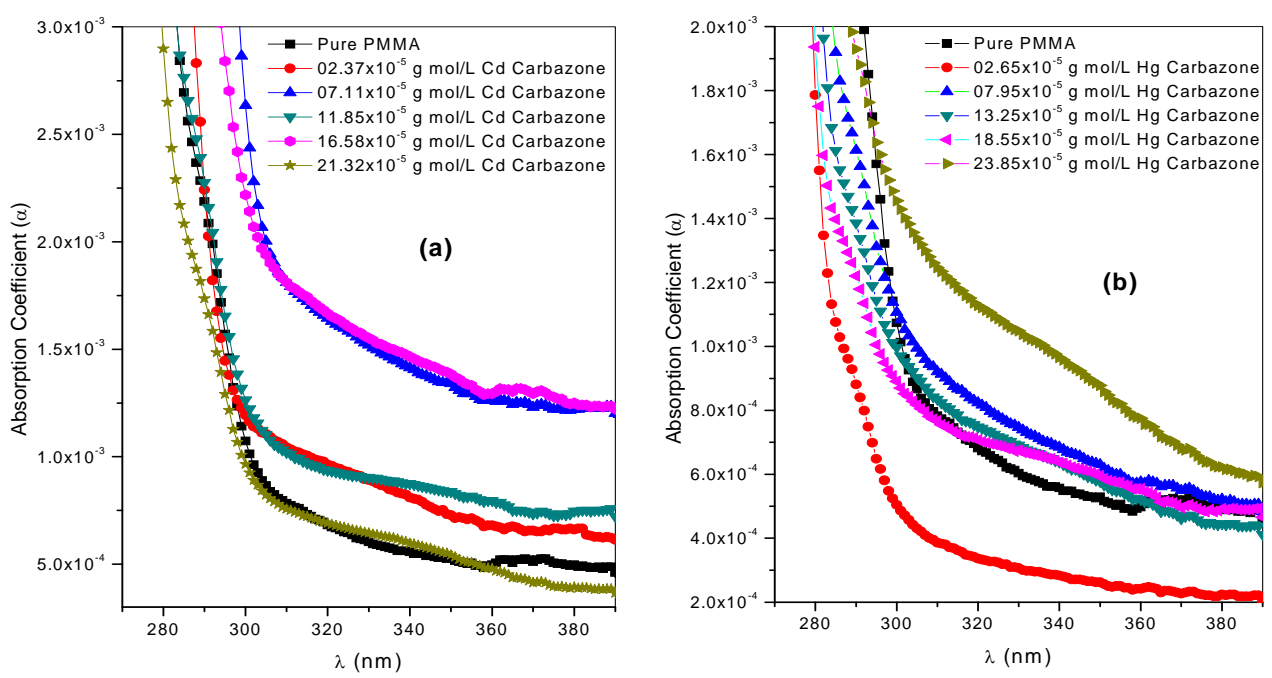

Fig. (5): Dependence of absorption coefficient $(\alpha)$ on wavelength in $\mathrm{nm}$ for methylmethacrylate polymerized in absence and presence of different concentrations of $\mathrm{Cd}(\mathrm{a})$ and $\mathrm{Hg}$ (b) carbazone. 


\subsection{Determination of the optical band gap and Urbach energy of the films}

The dependence of the absorption coefficient as a function of photon energy given by Davis and Mott [14] as

$$
\alpha h v=B\left(h v-E_{g}\right)^{n}
$$

where $\alpha$ is the absorption coefficient, $h v$ is the photon energy, factor $B$ depends on the transition probability and can be assumed to be constant within the optical frequency range, $E_{g}$ is the optical band gap, and the index $n$ is the power which characterizes the transition process. Both allowed direct $\left(n^{1 / 2}\right)$ and indirect $\left(\mathrm{n}^{2}\right)$ transitions were noticed by plotting $(\alpha h v)^{2}$ and $(\alpha h v)^{1 / 2}$ as a function of photon energy. The goodness of the fit was determined by the adjacent $\mathrm{R}^{2}$ which found to be 0.96809 and 0.9827 with average gap errors \pm 0.00066 and $\pm 0.0888 \mathrm{eV}$ for direct and indirect gap respectively in the case of Cd carbazone. Moreover, $\mathrm{R}^{2}$ found to be 0.98148 and 0.98523 with average gap errors \pm 0.00052 and $\pm 0.0743 \mathrm{eV}$ for direct and indirect gap respectively in the case of $\mathrm{Hg}$ carbazone. In amorphous systems the lake of translation symmetry allows to assume that the most probable transition is indirect. This is due to the fact that in such case the wave vector is not defined, i.e. it is not good quantum number. The dependence of $(\alpha)^{1 / 2}$ on incident energy in eV is shown in Fig. (6).
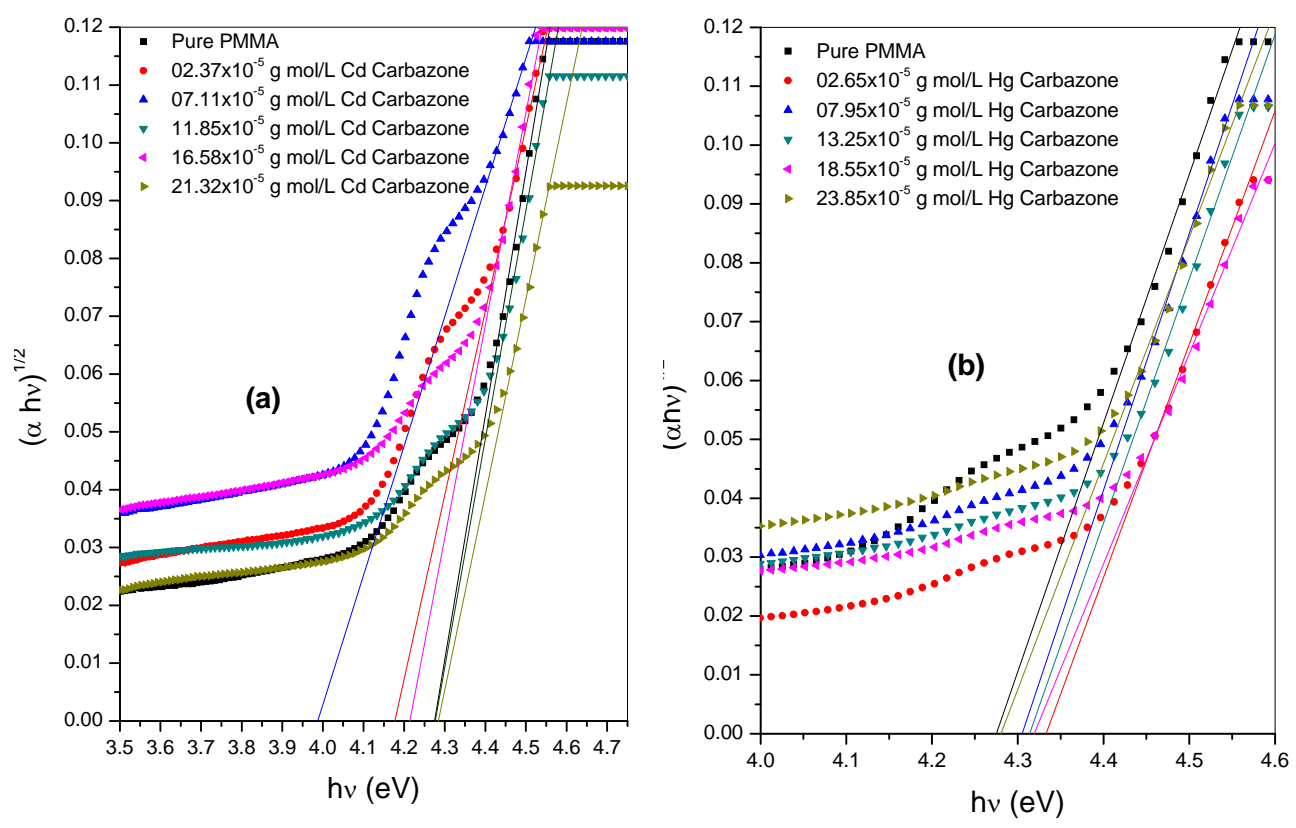

Fig. (6): Tauc plots of methylmethacrylate polymerized in absence and presence of absence and presence of different concentrations of $\mathrm{Cd}$ (a) and $\mathrm{Hg}$ (b) carbazone. 
In the range $\alpha(v) \leq 10^{4} \mathrm{~cm}^{-1}$, the absorption coefficient is commonly described by Urbach rule, which given by Urbach-Martienssen ${ }^{15}$ equation:

$$
\propto=\infty_{0} \exp \left(\frac{h v}{E_{v}}\right)
$$

where $\alpha_{0}$ is the pre-exponential factor, $h \nu$ is the incident photon energy, and $E_{\text {II }}$ is the Urbach energy which describe the width of the tails of the localized states in the gap and corresponding to the optical transition between localized states adjacent to the valence band and extended state in the conduction band which is lying above the mobility edge.

Taking the natural logarithm of this equation gives

$$
\ln \propto=\ln \alpha_{0}+\frac{h v}{E_{\sigma K}}
$$

Figure (7) represent plotting of $\ln \alpha$ against photon energy, the Urbach energy can be determined directly from the reciprocal of the slopes of the linear portion of these curves. This region is attributed to the electronic transition between a localized states. The origin of $E_{u}$ is related to the lattice disorder. The calculated values of the respective values of $E_{g}$ for indirect transitions together with the Urbach energy $E_{u}$ for the investigated system presented in Fig. (8.a, b).
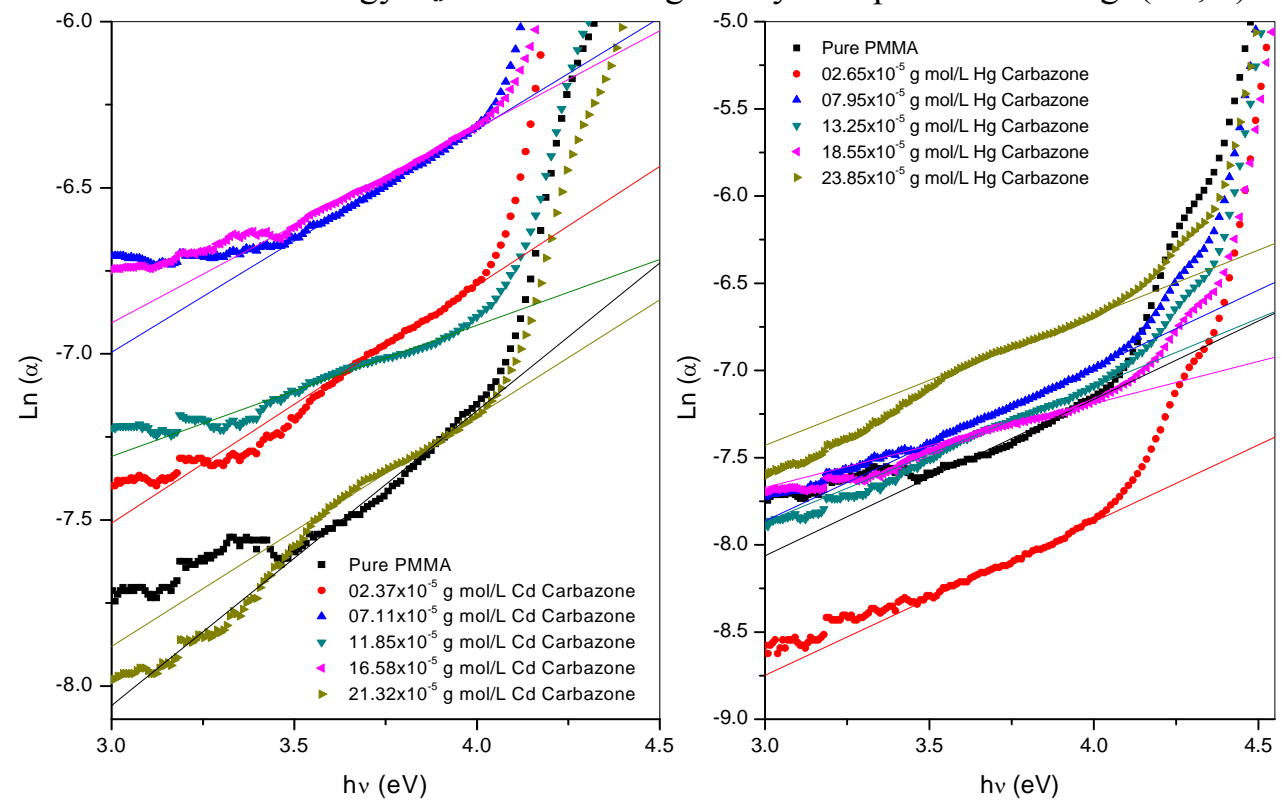

Fig. (7): Dependence of natural logarithm of $\alpha$ on photon energy for methylmethacrylate polymerized in absence and presence of different concentrations of $\mathrm{Cd}$ (a) and $\mathrm{Hg}$ (b) carbazone. 


\subsection{The Dispersion of the Refractive Index of the Films}

The dispersion of the refractive index plays an important role in the research for optical materials, because it is a significant factor in designing optical devices. According to the single-effective oscillator model proposed by Wemple and DiDomenico ${ }^{16}$ the optical data can be described to an excellent approximation by the relation:

$$
n^{2}=1+\frac{E_{d}^{2} E_{0}^{2}}{E_{0}^{2}-E^{2}}
$$

where $E_{0}$ is the single-effective oscillator energy, $E_{d}$ is the dispersion energy which is a measure of the average strength of the inter-band optical transitions and $\mathrm{E}$ is the photon energy. The latter is related to the coordination number of given atom. This model describes the dielectric response for transitions below the optical gap and plays an important role in determining the behavior of the refractive index [17].

To calculate the refractive index of the films under investigation. The following relation was used

$$
n=\frac{1-R(\lambda)}{1+R(\lambda)-2 \sqrt{R(\lambda)} \cos (\theta(\lambda))}
$$

where $R(\lambda)$ is reflectance, which calculated from conservation of energy, and $\theta(\lambda)$ is the angle of incidence.

The dependence of $E_{g}, E_{U}, E_{0}, E_{d}$ and percentage of conversion with respect to the complex concentration was presented in Fig. (8), where the percentage of conversion taken from other work $^{11}$ which presented here for comparison. The reduction in the optical band gap (8.a) in the case of adding $\mathrm{Cd}$ up to $7.11 \times 10^{-5}$ g.mol/L, is attributed to cross - linking and increase of disorder of the PMMA occurred by doping.

The width of the localized states available in the optical band gap (the Urbach tail) affects the gap structure, which is related directly to a similar exponential tail for the density of states. Increasing the density of localized states in the band gap reveals increasing the number of unsaturated complex. Maximum activity can be noticed at $11.85 \times 10^{-5}$ and $18.55 \times 10^{-5} \mathrm{~g} \cdot \mathrm{mol} / \mathrm{L}$ for $\mathrm{Cd}$ and $\mathrm{Hg}$ respectively where high density of localized states in broad band gap was recorded. This may be attributed to the effect of internal potential fluctuation associated with the structural disorder. Then it deactivates at higher concentrations due to an apparent crowding of its higher population and confirm 
the creation of new active centers which are produced during the polymerization process. This leads to randomness increase and subsequently decreasing the order of the polymeric chain packing [11].

Dipole energy and dipole strength increase with increasing Cd carbazone content till that peak of maximum activity at a concentration $11.85 \times 10^{-5}$ g.mol/L, then it decrease confirming the creation of new localized states. The behavior in the case of cadmium differs from the behavior in the case of $\mathrm{Hg}$, where no significant changes were noticed for $\mathrm{E}_{0}$ or $\mathrm{E}_{\mathrm{d}}$ with increasing $\mathrm{Hg}$ concentration, which can be attributed to the high ionization potential of $\mathrm{Hg}$ compared with that of $\mathrm{Cd}(10.434 \mathrm{eV}$ for $\mathrm{Hg}$ and $8.991 \mathrm{eV}$ for $\mathrm{Cd})$ that make it difficult to produce a dipole moment and hence chemical bonds in the case of $\mathrm{Hg}$.

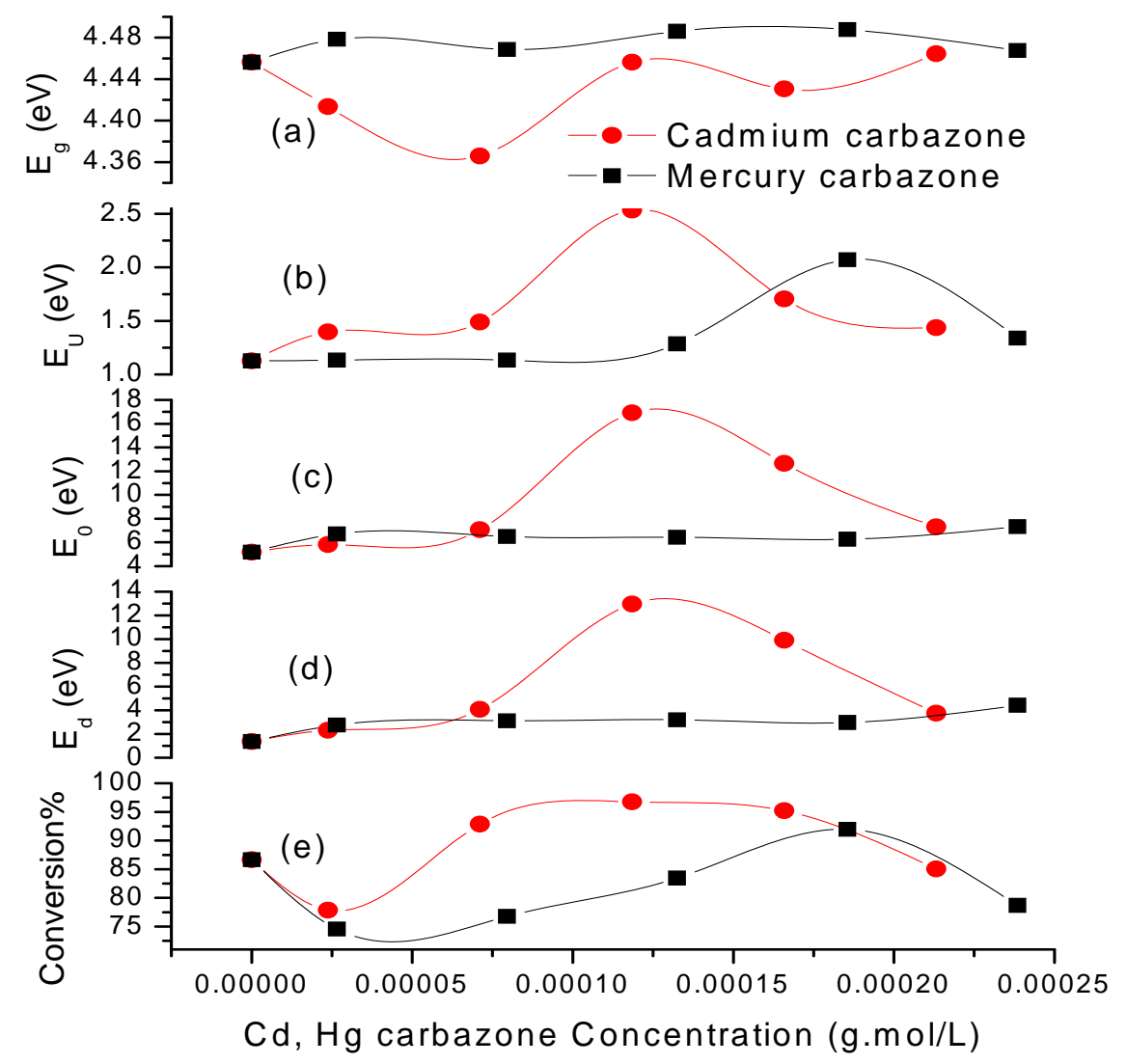

Fig. (8): Dependence of optical band gap (a), Urbach energy (b), single-effective oscillator energy (c), average strength of the inter-band optical transitions (d) together with percentage of conversion (e) from El-Said H.El-Mosallamy [11] with respect to the complex concentration 


\section{Conclusion}

Effect of using $\mathrm{Cd}$ and $\mathrm{Hg}$ carbazone complex as an additive through the polymerization of methylmethacrylate on the optical properties of poly methylmethacrylate sheets have been studied. Optical band gap $\mathrm{E}_{\mathrm{g}}$, width of the tails of the localized states $\mathrm{E}_{\mathrm{U}}$ and refractive index dispersion parameters, single-effective oscillator energy $E_{0}$, dispersion energy $E_{d}$, were calculated. Optical absorption edge in UV region broadened and exhibit red shift with addition of metal complex up to a certain concentration, and fluctuates with increasing concentration above this value. These observations indicates the conjugation of bonds in the studied region and fluctuation in the internal potential associated with the structural disorder.

\section{References}

1. D. Dorranian, Z. Abedini, A. Hojabri, M. Ghoranneviss, Journal of NonOxide Glasses, Vol. 1, No. 3, September, 217 (2009).

2. Hailin Hu, P.K. Nair, Surface and Coatings Technology, 81, 183 (1996).

3. J. E. Klmberg-Sapieha, L. Martinu, N. L. S. Yamasaki and C. W. Lantman, Thin Solid Films, 476(1), 101 (2005).

4. H.M. Zidan, M. Abu-Elnader, Physica, B 355, 308 (2005).

5. K. Beev, K. Temelkov, N. Vuchkov, T. Petrova, V. Dragostinova. R. Stoycheva-Topalova, S. Sainov and N. Sabotinov, Journal of Optoelectronics and Advanced Materials, 7 (3), 1315 (2005).

6. X. M. Dong, Y. Luo, L. N. Xie, R. W. Fu and M. Q. Zhang, Thin Solid Films, 516, 7886 (2008).

7. P. Poornesh, P. K. Hedge, G. Umesh, M. G. Manjunatha, K.B. Manjunatha and A. V. Adhikari, Optics \& Laser Technology, 42, 230 (2010).

8. G. Kim, European Polymer Journal, 41(8), 1729 (2005).

9. N. G. McCrum, C. P. Buckley, C. B. Bucknall; "Principles of polymer engineering", $2^{\text {nd }}$ Edition, Oxford Scientific Publications, Oxford, (1999).

10. A.A.Gridnev, S.D. Ittel; Chem. Rev., 101, 3611 (2001).

11. El-Said H.El-Mosallamy, Macromolecules:An Indian Journal, 9, 1 (2013).

12. Tony Owen, "Fundamentals of modern UV-visible spectroscopy" Agilent Technologies, Germany (2000).

13. Z.M. Elimat, A.M. Zihlif, G. Ragosta, Physica, B 405, 3756 (2010).

14. E.A. Davis and N.F. Mott, Phil. Mag., 22, 903 (1970).

15. Manal Abdel-Baki, Fouad El-Diasty b, Fathy A. Abdel Wahab, Optics Communications, 261, 65 (2006).

16. S. H. Wemple and M. Jr. DiDomenico, Phys. Rev., B3, 1338 (1971).

17. Michael Herrmann, Frank Platte, Konstantinos Nalpantidis, René Beigang, H. Michael Heise, Vibrational Spectroscopy, 60, 107 (2012). 